\title{
Energy-efficient autonomous solar water-pumping system for permanent magnet synchronous motors
}

\author{
Riccardo Antonello, Member, IEEE, Matteo Carraro, Alessandro Costabeber, Member, IEEE, Fabio Tinazzi, and \\ Mauro Zigliotto, Member, IEEE
}

\begin{abstract}
This paper presents a novel stand-alone solarpowered water pumping system, especially suited for usage in rural or remote areas. The system is primarily designed to reduce both cost and complexity, while simultaneously guaranteeing optimal utilisation of the photovoltaic generator. The use of standard hardware and control architectures ensures ease of installation, service and maintenance. The proposed solution consists of a water pump driven by a permanent magnet synchronous motor (PMSM), controlled by a conventional field oriented control scheme. The photovoltaic array is directly connected to the DC bus of the inverter, with no intermediate power conversion stages. A perturbation based extremum-seeking controller adjusts the motor speed reference to attain the maximum power point operation of the photovoltaic array. Both simulations and experimental results on a full-scale prototype support the effectiveness of the proposed system.
\end{abstract}

Index Terms-PMSM drives, MPPT tracking, PV water pumping, extremum seeking control

\section{NOMENCLATURE}

Variables names convention (letter/subscript):

- uppercase/uppercase: large-signal DC quantity.

- lowercase/lowercase: small-signal quantity.

- uppercase/lowercase: amplitude of a small-signal sinusoidal perturbation.

- lowercase/uppercase: total quantities (i.e. small-signal perturbation superimposed on large-signal DC quantity)

Symbols used:

$\begin{array}{ll}p_{P}, u_{P} & \text { PV array output power and voltage. } \\ p_{C}, p_{I} & \text { power stored in DC bus capacitor, power } \\ & \begin{array}{l}\text { absorbed by the inverter. } \\ p_{R}, p_{M}\end{array} \\ \begin{array}{l}\text { Joule losses and delivered mechanical } \\ \text { power. }\end{array} \\ \begin{array}{l}\text { PMSM voltages and currents in the } d q \\ \text { frame. }\end{array}\end{array}$

Manuscript received March 4, 2016; revised May 26, 2016;

R. Antonello, F. Tinazzi, and M. Zigliotto are with the Department of Management and Engineering, University of Padova, 35131 Padova, Italy (e-mail: riccardo.antonello@unipd.it; fabio.tinazzi@unipd.it; mauro. zigliotto@unipd.it).

M. Carraro is with Sael Srl, 36040 Torri di Quartesolo, Italy (e-mail: carraro@sael.it).

A. Costabeber is with the Department of Electrical and Electronic Engineering, University of Nottingham, Nottingham, NG7 2RD, U.K. (e-mail: Alessandro.Costabeber@nottingham.ac.uk).

$$
\begin{array}{ll}
\omega_{M}, a_{M} & \text { PMSM speed and acceleration. } \\
i_{D, Q}^{*}, \omega_{M}^{*}, a_{M}^{*} & \text { current/speed/acceleration references. } \\
\tau_{M}, \tau_{F}, \tau_{L} & \text { motor, friction and load (pump) torques. } \\
B_{0, F}, B_{0, F} & \text { coefficients of the friction torque vs speed. } \\
B_{0, L}, B_{0, L} & \text { coefficients of the load torque vs speed. } \\
U_{p}, \omega_{u}, \varphi_{u} & \text { perturbation in the DC bus - amplitude, } \\
& \text { frequency and phase. }
\end{array}
$$

Additional symbols are defined in Tab. I and II.

\section{INTRODUCTION}

$\mathbf{W}$ ATER provision in remote or isolated areas lacking of water and energy distribution systems has always been a challenge [1]. In those regions, typically desert or mountainous, that are not easily accessible by conventional transportation means, water pumping represents the only viable method to ensure a minimum water supply even during severe droughts. Hand pumps, possibly operated with the assistance of animals, are only suitable for low consumption rates and pumping heads. On the other hand, mechanically operated pumps can sustain all those activities, besides of conventional domestic uses, that are typical of a small rural community, such as irrigation and livestock. Most of them are powered by diesel engines; however, especially for small communities in developing countries, their use could be impracticable because of the limited availability and high cost of fuel. Recently, the advent of cheaper photovoltaic (PV) panels and mass produced pumps has made solar powered water pumping a viable and competitive solution [2], especially in those areas interested by the presence of a large solar radiation [3]. Solar pumping systems offer many advantages over the more traditional diesel pumps, including improved reliability and reduced operational and maintenance costs. The availability of pumping power in these systems is well matched to the water demand, which is in fact largest at daytime. However, it is also affected by weather and environmental conditions, which are by their nature variable. In particular, intermittent power shortages could easily occur, especially in a cloudy day. In order to maximise the energy utilisation and thus reduce the breakeven point with respect to other solutions (e.g. diesel pumps), the PV source should be always operated at its point of maximum efficiency (i.e. Maximum Power Point - MPP). This prevents oversized PV panels, to the benefit of the overall system cost and reliability. A first example is reported in [4], where the MPP is found by shifting the PV array voltage by changing the motor speed in a predefined range and by measuring the inverter input 
power. Unfortunately, the technique suffers of the interference of sunshine variations and a certain complexity in fixing the range of speed sweep. The design of an energy efficient solar pumping system requires to match the motor-pump load curve with the maximum power point locus (on the power vs voltage curve) of the PV source. For small systems, based on DC motors, a DC/DC converter between the PV source and the converter is always inevitably required, since a perfect match is difficult to obtain through a direct connection. A good example is reported in [5], that uses an hybrid buck/buckboost DC/DC for a low-power PV pumping application. The power converter is obviously unavoidable when using $\mathrm{AC}$ motors. In [6], [7] an additional DC/DC converter is used to boost the DC bus voltage of the induction motor drive. This solution enables operation at higher speeds, but at the expense of an increased circuit complexity. In [8], a single stage solution is proposed, where an open-end winding induction machine is driven by a dual three-phase converter. This reduces voltage ratings but increases the components count in the conversion system. In general, the design of a stand-alone solar pumping system should guarantee maximum operating efficiency, by foreseeing the implementation of a suitable MPP tracking scheme. A comprehensive review of MPP trackers (MPPTs) for stand-alone PV systems can be found in [9][12]. In this paper, a novel stand-alone solar pumping system with a isotropic PMSM is presented. The speed reference is continuously adjusted in the direction that implies an increased power demand to the PV array. This action is performed by an ad-hoc MPPT that operates according to the extremum seeking control principle [13]-[17], which recalls the Ripple Correlation (RC) scheme [18]. The main difference consists on how the perturbation of the DC bus voltage, required to steer the working point toward the MPP, is generated. Rather than using the intrinsic voltage ripple of the switched power supply, as done in the ripple correlation scheme, in the proposed solution an artificial ripple is generated by purposely injecting a sinusoidal current perturbation in the PMSM. The paper is organised as follows. The architecture of the proposed MPPT is introduced in Sec. II, along with the basic modelling required to understand its working principle. The fundamental dynamics of the PV power regulation process is analysed in Sec. III, with an emphasis on the determination of the stable operating points. The control scheme is described in Sec. IV. The proposed solution has been tested both by simulations and on a full-size prototype, consisting of a $1 \mathrm{~kW}$ PV array plus a $5 \mathrm{~kW}$ PMSM that drives a virtual load pump, obtained by a $3.7 \mathrm{~kW}$ induction motor. The results are reported in Sec. V and Sec. VI. Final conclusions and remarks are reported in Sec. VII.

\section{System ARCHITECTURE AND BASIC MODELLING}

\section{A. System architecture overview}

The architecture of the proposed MPPT for a stand-alone solar-powered water pumping system is reported in Fig. 1. The water pump is driven by an inverter-fed PMSM, controlled with a conventional Field Oriented Control (FOC) scheme. The PV array is directly connected to the DC bus of the VSI,

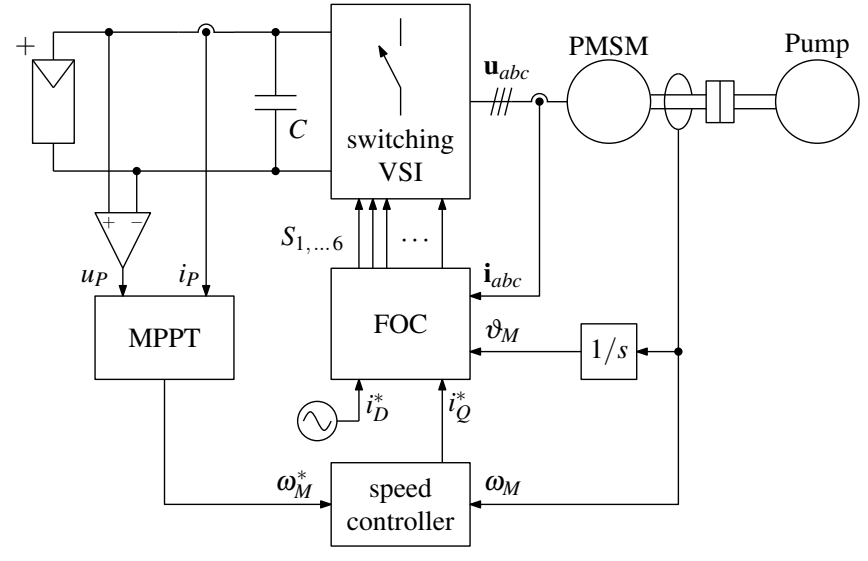

Fig. 1. The proposed stand-alone solar-powered water pumping system.

without any intermediate power conversion stage. The MPPT operates by adjusting the speed reference of the PMSM speed control loop, in order to draw the maximum available power, for any given irradiance condition.

\section{B. Power balance}

The PV power regulation capability of the proposed system can be better understood by resorting to its underlying instantaneous power balance equation. At the DC bus side of the inverter, it holds that

$$
p_{P}=p_{C}+p_{I}
$$

where $p_{P}, p_{C}$ and $p_{I}$ are the instantaneous powers delivered by the PV source, stored in the DC bus capacitor, and absorbed by the inverter, respectively. For what regards $p_{P}$, it is sufficient to mention that it is a function of the PV array voltage $u_{P}$, and that it has a single peak (MPP) under uniform solar irradiance conditions (see Fig. 2a), provided that all the PV panels in the array are perfectly matched [19]. As for $p_{C}$, it holds that

$$
p_{C}=C u_{P} \frac{d u_{P}}{d t}
$$

Regarding $p_{I}$, this is equal to the power delivered to the motorpump, if the inverter is assumed to have $100 \%$ efficiency, i.e.

$$
p_{I}=(3 / 2)\left(u_{D} i_{D}+u_{Q} i_{Q}\right)
$$

where the right-hand side of (3) is the instantaneous electrical power absorbed by the PMSM, expressed in the $d q$ reference frame synchronous to the rotor permanent magnet. The mechanical torque balance equation is given by

$$
\tau_{M}=J \frac{d \omega_{M}}{d t}+\tau_{F}+\tau_{L}
$$

where $J$ is the motor-pump total inertia and $\tau_{M}$ is the electromagnetic torque delivered by the PMSM. For an isotropic motor as that used in the paper, it is:

$$
\tau_{M}=(3 / 2) p \lambda_{M G} i_{Q}
$$

The friction torque $\tau_{F}$ generally includes a constant term due to dry friction $B_{0, F}$, and a term proportional to the speed due to ventilation. The load torque $\tau_{L}$, exerted by the pump, 
depends on the pump type (e.g. centrifugal, progressive cavity, etc. [1]). If the case of a centrifugal pump is considered, the two torque components are then:

$$
\tau_{F}=B_{1} \omega_{M}+B_{0, F} \quad \tau_{L}=B_{2} \omega_{M}^{2}+B_{0, L}
$$

By first substituting (6) in (4) and then considering a steady state condition $\left(\omega_{M}=\Omega_{M}\right)$, so that the inertial torque can be neglected, the mechanical power balance becomes:

$$
P_{M}\left(\Omega_{M}\right)=\tau_{M} \Omega_{M}=B_{2} \Omega_{M}^{3}+B_{1} \Omega_{M}^{2}+B_{0} \Omega_{M}
$$

where $B_{0}=B_{0, L}+B_{0, F}$. The quadrature current $I_{Q}$ is readily obtained from (5) and (7):

$$
I_{Q}\left(\Omega_{M}\right)=\frac{P_{M}\left(\Omega_{M}\right)}{(3 / 2) p \lambda_{M G} \Omega_{M}}
$$

while the FOC condition implies $I_{D}=0$, so that the Joule power losses at steady state are $P_{R}=3 / 2 R I_{Q}^{2}$. The power absorbed by the VSI, i.e.

$$
P_{I}=P_{I}\left(\Omega_{M}\right)=P_{R}\left(\Omega_{M}\right)+P_{M}\left(\Omega_{M}\right)
$$

is a monotonic function of the motor speed. The speed is then eligible as control variable to get the MPP condition.

\section{Limitation of the VSI absorbed power}

There are two factors limiting the power $p_{I}$ that the Voltage Source Inverter (VSI) can draw from the PV source. Namely, they are the PMSM nominal power $\left(P_{N}\right.$, Fig. 2a) and the voltage saturation limit of the VSI, which in turn depends on the DC bus voltage $u_{P}$. If the operations are carried out within the VSI linear modulation range, then the voltage vector lies always within the circle

$$
u_{D}^{2}+u_{Q}^{2}=\left(u_{P} / \sqrt{3}\right)^{2}
$$

For any given voltage $u_{P}$, the condition (10) sets a bound on the maximum achievable motor speed, and hence, because of (9), also on the maximum power request of the VSI. In fact, under full or quasi steady state conditions, the PMSM electrical balance equations reduce to

$$
U_{D}=-p \Omega_{M} L I_{Q}, \quad U_{Q}=R I_{Q}+p \Omega_{M} \lambda_{m g}
$$

with $I_{Q}$ given by (8). After replacing (11) within (10) and then solving for the motor speed, the upper bound $\bar{\Omega}_{M}\left(U_{P}\right)$, which depends on the voltage $U_{P}$, is found. This, in turn, can be replaced within (9), to obtain the maximum power $\bar{P}_{I}\left(U_{P}\right)$ that the VSI can request with a given DC bus voltage $U_{P}$. A closed form expression for the function $\bar{P}_{I}\left(U_{P}\right)$ is in general unavailable, but it can be evaluated numerically, once the nominal parameters of the PMSM and load are provided. For a load curve of the type (7), the function has a trend similar to that reported in Fig. 2a (dashed line). The shaded area in figure represents the unfeasible operating region, due to the $\bar{P}_{I}\left(U_{P}\right)$ constraint. The PV array and the motor-pump are properly matched when the MPP locus lies entirely within the feasible region. In such case, regardless of the solar irradiance condition, the MPP can always be achieved through a suitable adjustment of the motor speed.

\section{SYSTEM DYNAMICS FUNDAMENTALS}

The regulation of the PV power $p_{P}$ is governed by the dynamic equation (1). When the DC bus capacitor is present, an increased PV power absorption $p_{P}$ can be produced with either an increased or a decreased power demand $p_{I}$, depending on the position of the working point with respect to the MPP in the power vs. voltage plane. This aspect is better clarified with the aid of Fig. 2b and Fig. 2c. Consider first the case of an initial working point located on the right of the MPP, as the point (1) in Fig. 2b. Since the power demand $p_{I}$ at the given working point exceeds the PV power $p_{P}$, the extra power needed to satisfy the demand must be provided by the capacitor, meaning that $p_{C}<0$. Then, because of (2), the voltage $u_{P}$ drops until the new working point (2) is reached, where all the power demand is satisfied by the PV source. On the contrary, if the initial working point is between (2) and the MPP, the power provided by the PV source exceeds that demanded by the VSI. In this case, the voltage rises, again moving the working point toward (2). Therefore, the working point (2), as any other located on the right of the MPP, is a stable equilibrium point. To move it toward the MPP, it is sufficient to increase $p_{I}$, by increasing the motor speed.

The case of an initial working point located on the left of the MPP is shown in Fig. 2c. In (3), the power demand in excess of the PV power $p_{P}$ is provided by the capacitor, and then the voltage $u_{P}$ starts to drop. The working point moves to the left of (3) along the $p_{I}$ line, until the boundary of the unfeasible region is reached at point (4). Then, as the voltage $u_{P}$ further drops, the working point slides along the boundary of the unfeasible region, and $p_{I}$ starts to fall, i.e. the PMSM decelerates. A stable equilibrium is reached when the working point reaches the point (5). Here, any further voltage drop would produce an excess of PV power over the VSI demand, which in turn recharges the capacitor and brings the working point back to (5). The drive remains in (5) until the speed reference is reduced below $\bar{P}_{I}\left(U_{P}\right)$. In that case the VSI exits from saturation and (being $p_{P}>p_{I}$ ) the DC bus capacitor quickly recharges, until the corresponding stable equilibrium point at the right of the MPP is reached.

\section{MAXixum Power PoInt TRaCKING}

The MPPT automatically seeks the maximum power condition by continuously changing the motor speed in the direction that yields an increased power demand to the PV. To detect whether the current operating point is located either on the left or right side of the MPP, one can rely on the sign of the local derivative of the power vs voltage function $p_{P}\left(u_{P}\right)$ in the current operating point. The derivative can be estimated by inducing a small sinusoidal voltage perturbation $u_{p}=U_{p} \sin \left(\omega_{u} t+\varphi_{u}\right)$ on the DC bus, and then performing a correlation with the PV power perturbation that it generates. The generated power $p_{P}\left(u_{P}\right)$ can be approximated by its linearisation around the voltage $U_{P}$, yielding

$$
p_{P}\left(U_{P}+u_{p}\right) \approx P_{P}+p_{p}=p_{P}\left(U_{P}\right)+\delta\left(U_{P}\right) u_{p}
$$

where $\delta\left(U_{P}\right)=\partial p_{P} /\left.\partial u_{P}\right|_{U_{P}}$ is the local derivative of $p_{P}$ evaluated at $U_{P}$. This can be extracted from the amplitude 


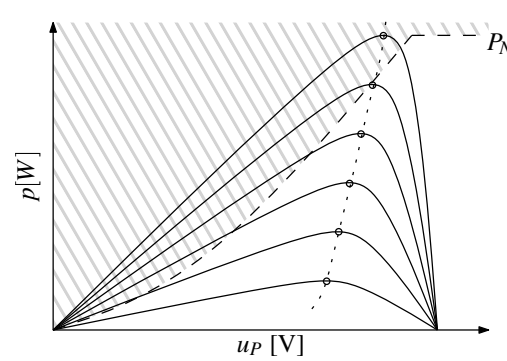

(a)

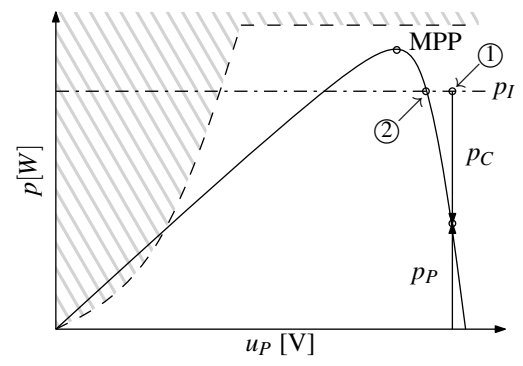

(b)

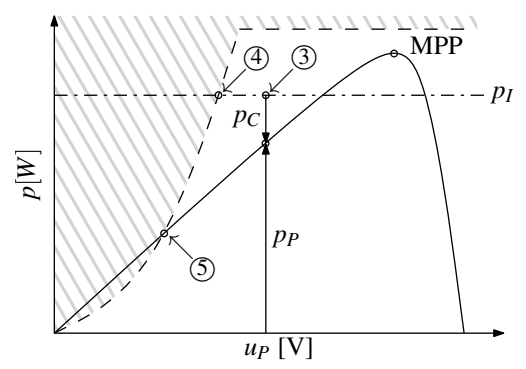

(c)

Fig. 2. PV power regulation details. (a) Region of feasible working points. (b)-(c) Dynamic behaviour on the right and left of the MPP.

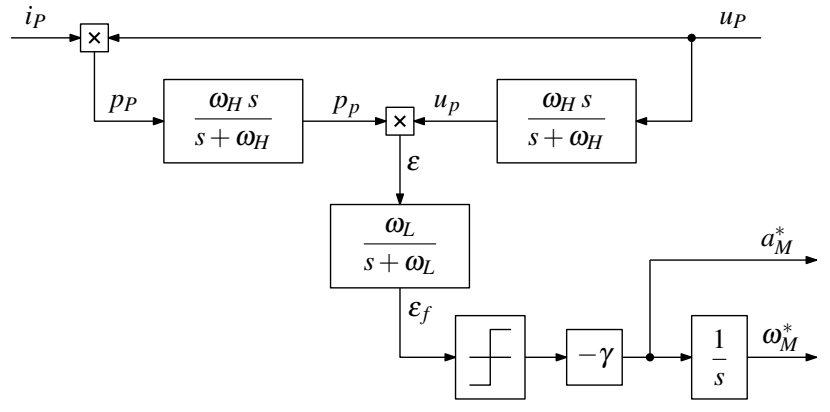

Fig. 3. Block diagram of the proposed extremum-seeking MPPT.

of the PV power perturbation $p_{p}$ by using the demodulation scheme shown in Fig. 3. Two high-pass filters are used to extract the alternating components $u_{p}$ and $p_{p}$ out of the PV voltage $u_{P}$ and power $p_{P}$. These are then combined with a mixer, to produce the error signal

$$
\varepsilon=\delta\left(U_{P}\right) u_{p}^{2}=\delta\left(U_{P}\right) U_{P}^{2} \frac{1-\cos \left(2 \omega_{u} t+2 \varphi_{u}\right)}{2}
$$

A low-pass filter is used to block the spurious component at twice the perturbation frequency in (13). The remaining DC component $\varepsilon_{f}$ is directly proportional to the local derivative $\delta\left(U_{P}\right)$. The sign of $\varepsilon_{f}$ is then used to adjust the PMSM speed reference $\omega_{M}^{*}$, in accordance with the rules outlined in Sec. III. In particular, $\omega_{M}^{*}$ is increased, at a fixed rate $\gamma$, when $\varepsilon_{f}<0$ and vice versa. The adjustment of the speed reference ends when $\varepsilon_{f}=0$, which is indeed the condition for MPP operation. Note that the aforementioned speed reference update law implicitly defines the acceleration reference signal

$$
a_{M}^{*}=-\gamma \operatorname{sign}\left(\varepsilon_{f}\right)
$$

that can be exploited as a feedforward compensation in the speed controller, to improve its response promptness.

\section{A. Generation of the voltage perturbation}

According to (1)-(2), a voltage perturbation is produced through a perturbation of the power $p_{I}$ absorbed by the inverter. This can be equally generated by a current perturbation injected either on the $d$ or $q$ axis (or both), as it can be noticed from (3). However, since the current $i_{Q}$ is related to the torque production (see (5)), and then a perturbation on it would induce an undesirable torque ripple, it is preferable to inject a small current perturbation $i_{d}=I_{d} \sin \left(\omega_{i} t\right)$ on the $d$ axis. At steady state, the currents $i_{D}=i_{d}$ and $i_{Q}=I_{Q}$ are generated by the voltages $u_{D}=U_{D}+u_{d}$ and $u_{Q}=U_{Q}+u_{q}$, where $U_{D} U_{Q}$ are defined by (11) and

$$
u_{d}=U_{d} \sin \left(\omega_{i} t+\varphi_{d}\right) \quad u_{q}=U_{q} \sin \omega_{i} t
$$

with

$$
U_{d}=I_{d} \sqrt{R^{2}+\left(\omega_{i} L\right)^{2}}, \quad U_{q}=p \Omega_{M} L I_{d}
$$

and $\varphi_{d}=\operatorname{atan}\left(\omega_{i} L / R\right)$. After replacing the previous expressions within (3), it follows that $p_{I}=P_{I}+p_{i}$, with $P_{I}=(3 / 2) U_{Q} I_{Q}$ and

$$
p_{i}=(3 / 4)\left(U_{d} I_{d}\right)\left[\cos \varphi_{d}-\cos \left(2 \omega_{i} t+\varphi_{d}\right)\right]
$$

In particular, the perturbation $p_{i}$ consists of a sinusoidal term pulsating at twice the frequency of the injected current perturbation, superimposed to a small offset. The voltage perturbation $u_{p}$ induced on the DC bus by $p_{i}$ can be determined by linearising (1)-(2) around the working point $\left(U_{P}, P_{I}\right)$ :

$$
C U_{P} \frac{d u_{p}}{d t}-\delta\left(U_{P}\right) u_{p}=-p_{i}
$$

The corresponding transfer function from the power perturbation input $p_{i}$ to the voltage perturbation output $u_{p}$ is

$$
G(s)=\frac{1}{\delta\left(U_{P}\right)-s C U_{P}}
$$

Note that the model is bounded input-bounded output (BIBO) stable only when $\delta\left(U_{P}\right)<0$, i.e. when the working point is located on the right of the MPP, confirming the intuitive reasoning performed in Sec. III. The AC component of the voltage perturbation is $U_{p} \sin \left(2 \omega_{i} t+\varphi_{u}\right)$, with

$$
U_{p}=\left|G\left(j 2 \omega_{i}\right)\right|(3 / 4)\left(U_{d} I_{d}\right) \quad \varphi_{u}=\varphi_{d}+\angle G\left(j 2 \omega_{i}\right)
$$

Nevertheless, as far as the working point is "sufficiently close" to the MPP, the only unstable pole of (19) is located very close to the imaginary axis, so that the system (18) can be classified as "moderately unstable", according to the definition provided in [20]. The same paper proves that the MPPT is capable of counteracting the natural instability of the system, at least in a small neighbourhood around the MPP, provided that the speed controller response is fast enough. In this sense, the proposed addition of the acceleration feedforward eqref eq: aM has proved of great benefit. If the transition around the MPP is too fast, the working point moves far away from the MPP, 
TABLE I

PV PANEL AND PMSM NOMINAL DATA

\begin{tabular}{lcc}
\hline Panel id & - & Azimut AZM 546/225P \\
Maximum power & - & $225 \mathrm{~W}$ \\
Tolerance on power & - & $0 \% \div 3 \%$ \\
Voltage at MPP & - & $27.78 \mathrm{~V}$ \\
Current at MPP & - & $8.10 \mathrm{~A}$ \\
Short circuit current & - & $8.65 \mathrm{~A}$ \\
Open circuit voltage & - & $33.76 \mathrm{~V}$ \\
\hline Nominal current & - & $12.1 \mathrm{~A}$ \\
Nominal speed & - & $3000 \mathrm{rpm}$ \\
Nominal torque & - & $16 \mathrm{Nm}$ \\
Pole pairs & $p$ & 3 \\
Stator resistance & $R$ & $0.35 \Omega$ \\
Stator inductance & $L$ & $12 \mathrm{mH}$ \\
PM flux linkage & $\lambda_{M G}$ & $0.28 \mathrm{Vs}^{2}$ \\
Rotor inertia & - & $0.0035 \mathrm{kgm}^{2}$ \\
Rotor inertia (with load motor) & $J$ & $0.024 \mathrm{kgm}^{2}$ \\
\hline
\end{tabular}

TABLE II

MPPT SETTINGS

\begin{tabular}{lcc}
\hline Current perturbation amplitude & $I_{d}$ & $0.25 \mathrm{~A}$ \\
Current perturbation frequency & $\omega_{i}$ & $250 \mathrm{~Hz}$ \\
HPF cut-off frequency & $\omega_{H}$ & $25 \mathrm{~Hz}$ \\
LPF cut-off frequency & $\omega_{L}$ & $2.5 \mathrm{~Hz}$ \\
Integrator gain & $\gamma$ & $25 \mathrm{rpm} / \mathrm{s}$ \\
Current control bandwidth \& phase margin & - & $250 \mathrm{~Hz}, 70^{\circ}$ \\
Speed control bandwidth \& phase margin & - & $5 \mathrm{~Hz}, 60^{\circ}$ \\
DC bus capacitor & $C$ & $3 \times 130 \mu \mathrm{F}$ \\
\hline
\end{tabular}

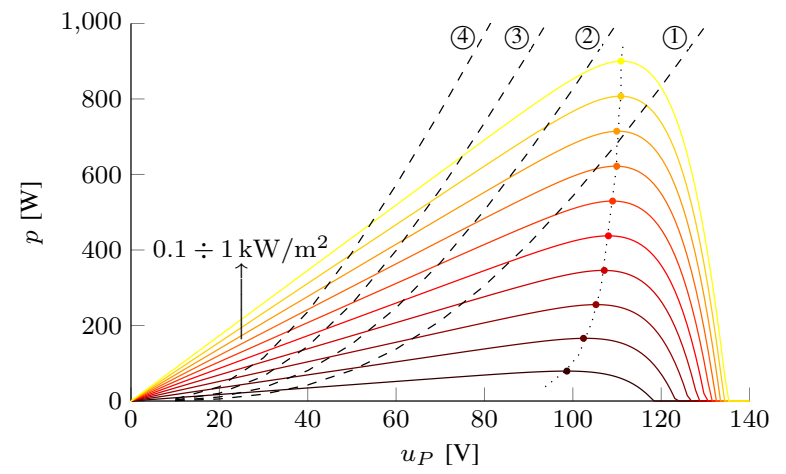

Fig. 4. Unfeasible region boundaries. Starting with (1), the values are 1, 2, 4 and 8 times the base value $B_{2}=2.5 \times 10^{-5} \mathrm{Nm} / \mathrm{rpm}^{2}$.

e.g. in a point like (3) in Fig. 2c. Spontaneously, it would move towards the point (5), but as the local derivative $\delta\left(U_{P}\right)$ is correctly detected by the perturbation-based method, the speed reference is reduced correspondingly and the MPP is soon reached again.

\section{Simulation RESUlts}

Accurate numerical simulations of the proposed MPPT were preformed both to validate the system dynamics analysis reported in the previous sections and to tune the controller parameters for the subsequent experimental tests. The nominal parameters and MPPT settings used for simulations are collectively reported in Tab. I and II.

\section{A. PV array characteristic and feasible operating regions}

The power vs voltage characteristics of the PV array at different solar irradiance levels $\left(100 \mathrm{~W} / \mathrm{m}^{2}\right.$ to $1 \mathrm{~kW} / \mathrm{m}^{2}$, in steps of $\left.100 \mathrm{~W} / \mathrm{m}^{2}\right)$ and the same temperature $\left(30^{\circ} \mathrm{C}\right)$ are shown in Fig. 4. It also reports the trend of the power limitation curves due to the VSI voltage saturation limit (dashed lines) when the pump has the quadratic load curve characteristic (6), for four different values of the coefficient $B_{2}(1,2,4$ and 8 times the base value $B_{2}=2.5 \times 10^{-5} \mathrm{Nm} / \mathrm{rpm}^{2}$ ). The curves have been determined with the procedure explained in Sec. II-C. For smaller values of the coefficient $B_{2}$, a larger pump speed, and hence a larger PMSM supply voltage (see (11)), are required to absorb a certain level of PV power. This implies that for a given PV voltage $u_{P}$, the VSI voltage saturation limit is reached at smaller power absorption levels, as clearly evident in Fig. 4. In particular, note that for the smallest value of $B_{2}$, the voltage saturation limit (curve (1)) does not allow to reach the MPP when the solar irradiance is greater than $\approx 800 \mathrm{~W} / \mathrm{m}^{2}$. On the other hand, for the other values of $B_{2}$, the MPP locus is completely contained in the feasible operating region. The simulation tests reported in the next sections are performed with a pump coefficient $B_{2}=5.0 \times 10^{-5}$, associated to the curve (2) in Fig. 4 .

\section{B. Convergence to the MPP}

Figure 5 illustrates the typical response of the MPPT under constant solar irradiance and temperature conditions $\left(900 \mathrm{~W} / \mathrm{m}^{2}\right.$ at $\left.30^{\circ} \mathrm{C}\right)$.

Without the acceleration feedforward compensation (Fig. 5b and $5 \mathrm{~d}$ ) the speed adjustments are not rapid enough to counteract the quick PV voltage drop occurring once the power absorption exceed the maximum available at the MPP. The working point quickly moves to the only stable equilibrium at the left of the MPP, located at the intersection of the VSI voltage saturation limit curve and the current power vs voltage characteristic (point (1) in Fig. 5d). The system remains there until the motor power demand (related to the motor speed) is brought below the power availability at the equilibrium point. Then, the working point quickly moves to the right of the MPP, to a stable operating point located at the same power level (point (2) in Fig. 5d). Once there, the MPPT starts to increase the speed again, and the whole cycle is repeated indefinitely, never reaching a stable MPP operating condition.

On the other hand, in presence of the proposed acceleration feedforward (Fig. 5a and 5c) the speed regulation is faster, so that once the MPP is reached, the controller is capable of contrasting, through rapid changes of the motor speed, the natural tendency of $u_{P}$ to quickly drop, which would cause a departure of the operating point from the MPP. It is worth to point out here that, in principle, a similar result could have been obtained by enlarging the speed control bandwidth. However, the presence of the measurement noise poses a limitation to the enlargement, so that the feedforward action is much more effective.

To complete the analysis, Fig. 6 illustrates the tracking performance of the MPPT (with acceleration feedforward) in presence of variations of the solar irradiance. A 20 s linear 

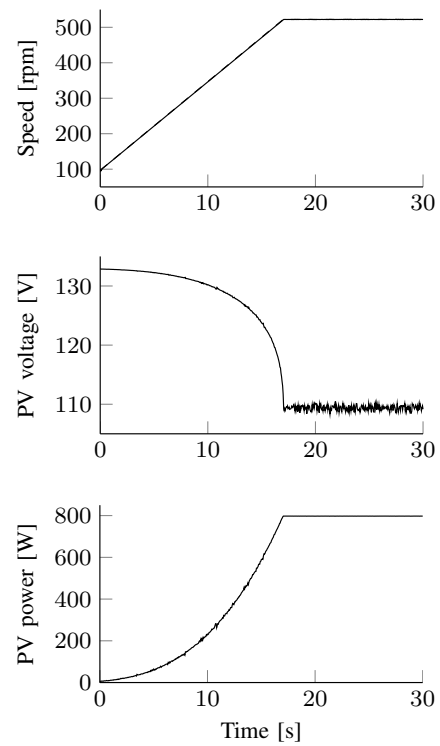

(a)
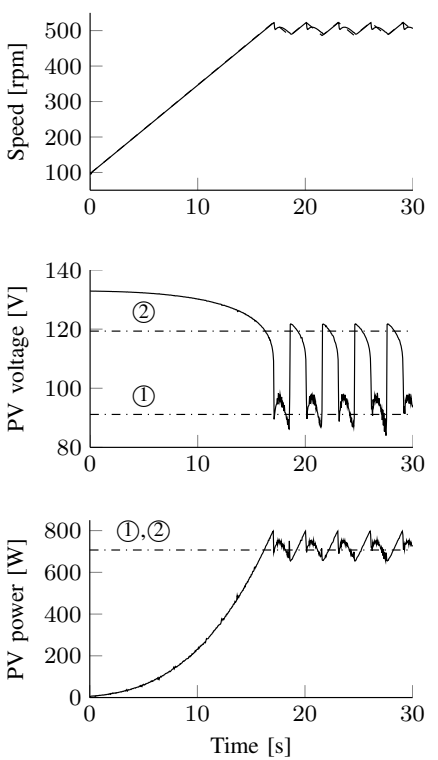

(b)

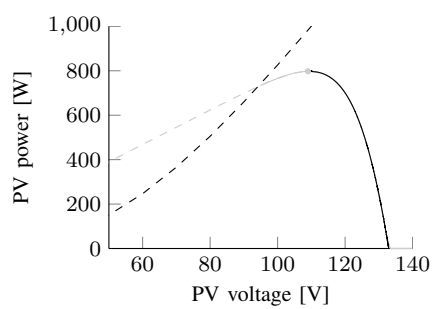

(c)

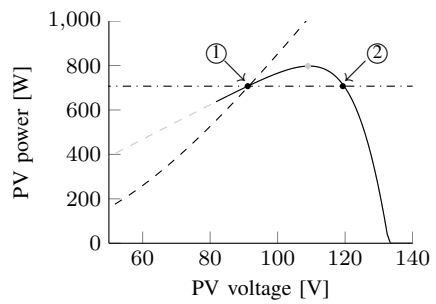

(d)

Fig. 5. MPPT response under constant solar irradiance conditions $\left(900 \mathrm{~W} / \mathrm{m}^{2}\right.$ at $\left.30^{\circ} \mathrm{C}\right)$, with [(a), (c)] and without [(b), (d)] acceleration feedforward.
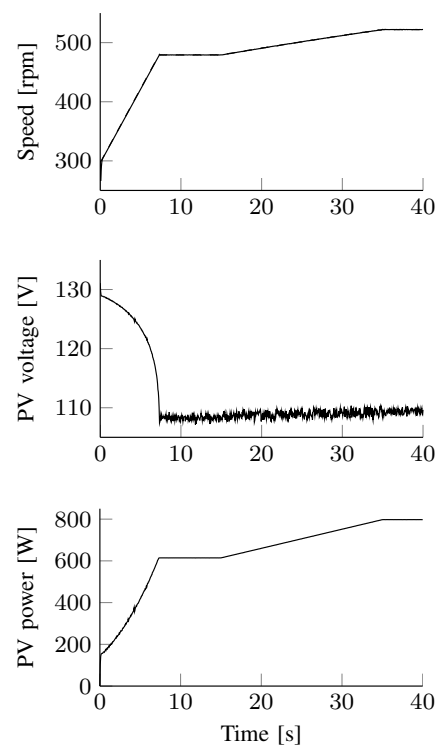

(a)
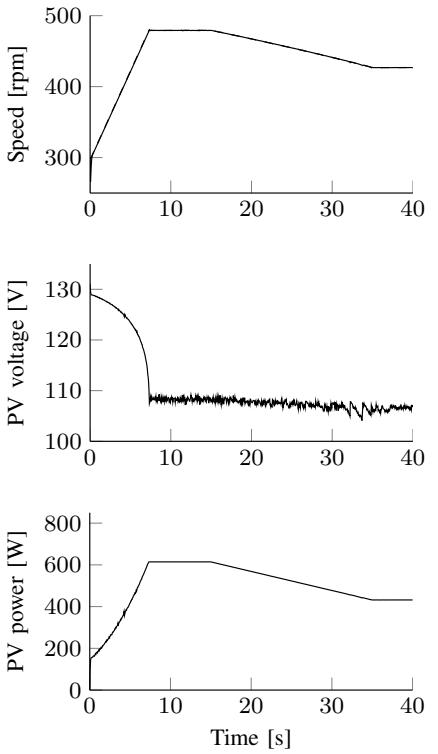

(b)

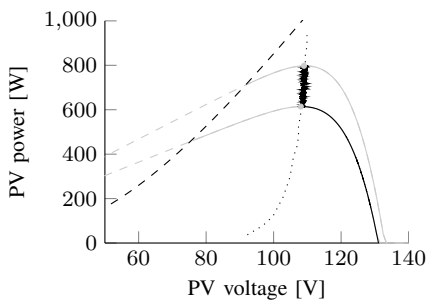

(c)

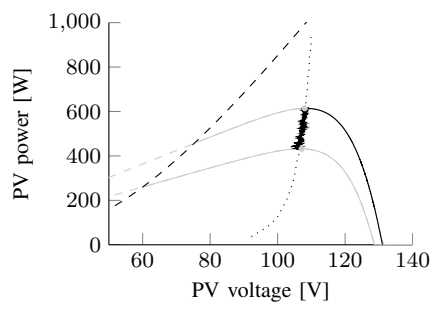

(d)

Fig. 6. MPPT response to variations of solar irradiance. (a), (c): $20 \mathrm{~s}$ ramp-up variation from $600 \mathrm{~W} / \mathrm{m}^{2}$ to $800 \mathrm{~W} / \mathrm{m}^{2}$ (starting at $15 \mathrm{~s}$ ); (b), (d): $20 \mathrm{~s}$ ramp-down variation from $600 \mathrm{~W} / \mathrm{m}^{2}$ to $400 \mathrm{~W} / \mathrm{m}^{2}$ (starting at $15 \mathrm{~s}$ ).

ramp variation (starting at $15 \mathrm{~s}$ ) is considered in the tests. The responses of Fig. $6 \mathrm{a}$ and $6 \mathrm{c}$ refer to a ramp-up from $600 \mathrm{~W} / \mathrm{m}^{2}$ to $800 \mathrm{~W} / \mathrm{m}^{2}$, while those of Fig. $6 \mathrm{~b}$ and $6 \mathrm{~d}$ are relative to a ramp-down from $600 \mathrm{~W} / \mathrm{m}^{2}$ to $400 \mathrm{~W} / \mathrm{m}^{2}$. In both cases, the MPP is properly reached after an initial transient, and continuously tracked while the solar irradiance is changing.

\section{EXPERIMENTAL RESULTS}

The full-size system prototype used for the experimental tests consists of a PV array (Fig. 7a), which provides the power supply to a PMSM (Fig. 7b). The nominal parameters of the PV modules and the PMSM are reported in Tab. I. The PMSM is connected to a $3.7 \mathrm{~kW}$ induction motor (IM) that emulates the mechanical load curve of a real pump. The power supply of the induction motor is derived from the grid. Two separated inverters are used for the motors. The PMSM VSI is fully controlled via a dSpace DS1104 board; the control sampling and PWM switching frequencies are both equal to $10 \mathrm{kHz}$. The IM drive implements a basic torque control loop, with the torque reference computed by the dSpace card. Fig. 8 reports the experimental response of the proposed MPPT under approximatively constant solar irradiance and temperature conditions (sunny day with temperature above $35^{\circ} \mathrm{C}$ ). Similarly 

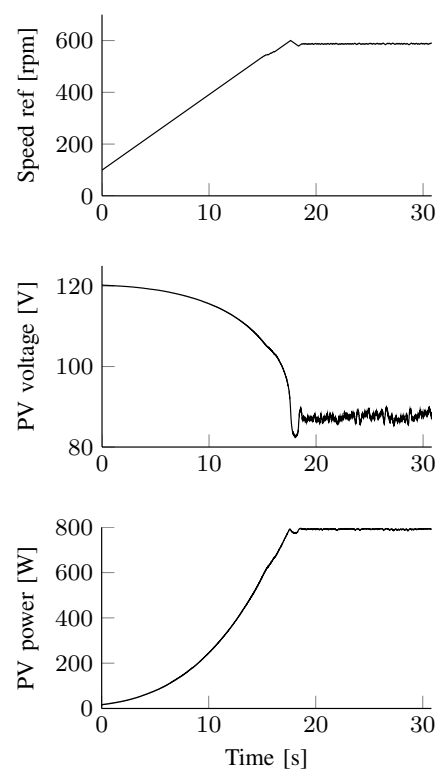

(a)
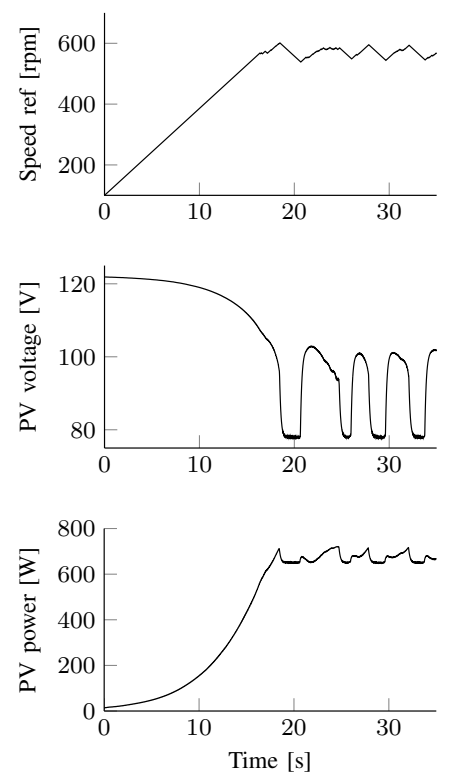

(b)

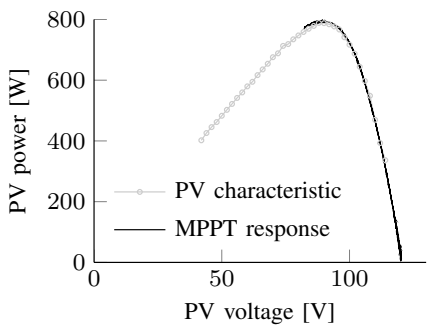

(c)

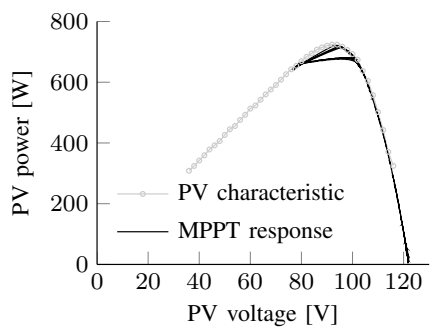

(d)

Fig. 8. Experimental results under approximatively constant solar irradiance and temperature conditions (sunny day with temperature above $35^{\circ} \mathrm{C}$ ). $($ a), $(\mathrm{c})$ : with acceleration feedforward compensation; (b), (d): without acceleration feedforward compensation.

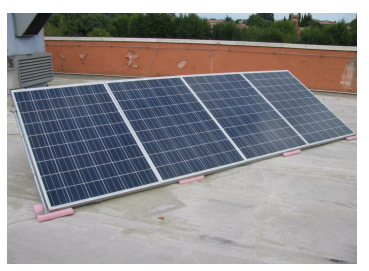

(a)

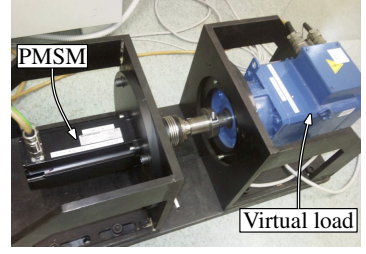

(b)
Fig. 7. Experimental setup. (a) PV array. (b) Motor test-bench.

to Fig. 5 of Sec. V, the tests are performed either by enabling or disabling the acceleration feedforward in the controller. The IM is programmed to emulate the same pump load curve characteristic considered for the simulation tests. The experimental results are very similar to the simulation ones. As expected, the MPPT is unable to guarantee stable operations at the MPP if the motor speed adjustments are not sufficiently fast. Fig. 9 reports the experimental $d-q$ currents and the relative phase currents during the same experiments described in Fig. 8, in presence of the acceleration feedforward. A zoomed detail of the phase currents at the end of the transient is reported in Fig. 9d.

\section{CONCLUSIONS}

A novel stand-alone solar-powered water pumping system based on a PMSM has been introduced in this paper. The system design operates at maximum efficiency, thanks to the implementation of a MPP tracking scheme. The theory of operations has been described by resorting to a power balance analysis, which allows to predict all the salient features of the overall system dynamics. The proposed mathematical approach allows a smart prediction of the feasible operating region in the PV power vs voltage plane, for any given pump load curve. Therefore, it represents an effective means to select the pump that best fits for the exploitation of a given PV array to its maximum efficiency. Several simulation and experimental tests have confirmed the validity of the proposal and the correspondence of the real system to the predictions that come from the power-balance based theory. The experiments have also put in evidence that the promptness of the speed regulation plays a crucial role for guaranteeing stable operations at the MPP. In this sense, the inclusion of an acceleration feedforward in the speed controller is beneficial for the purpose, giving the project a distinctive trait for a direct industrial implementation.

\section{A. Advantages and trends}

Nowadays, the vast majority of applications feature cheap inverter-fed induction motors (IM) with both rotor and stator in an oil bath. Motor and inverter are usually far from each other (from 20 to $200 \mathrm{~m}$ ). What is implicit in the present proposal is a step towards the use of more performing PMSM motors, fed by inverter integrated in the motor-pump system. This is particularly relevant in extreme climate conditions such as desert areas where the power converter cabinet has critical cooling requirements. Indeed, this configuration minimises the number of stages, simplifying manufacturing and installation. Also the diameter can be reduced, allowing narrower ground holes.

For sub-kW motors, the efficiency gap between PMSM and IM is of about $10 \%$. This yields considerable savings, in case of grid-connected plants. In case of stand-alone ones, a more meaningful comparison can be carried out in term of extra PV panels required for feeding the same pump. The cost of extra PV installation exceeds that of the PMSM vs IM, up to nominal powers of some kilowatts. For higher power, the 


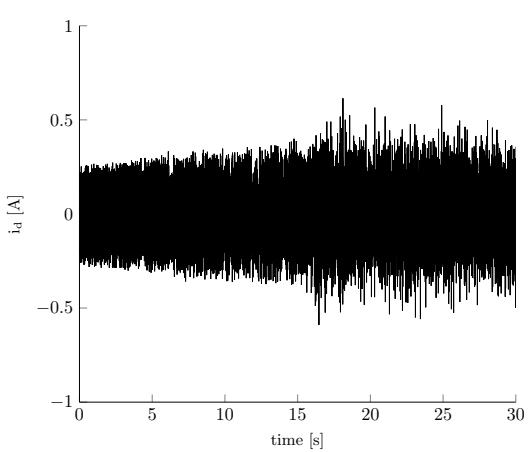

(a)

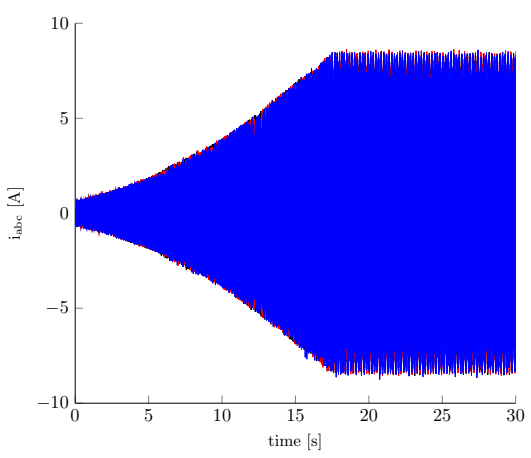

(c)

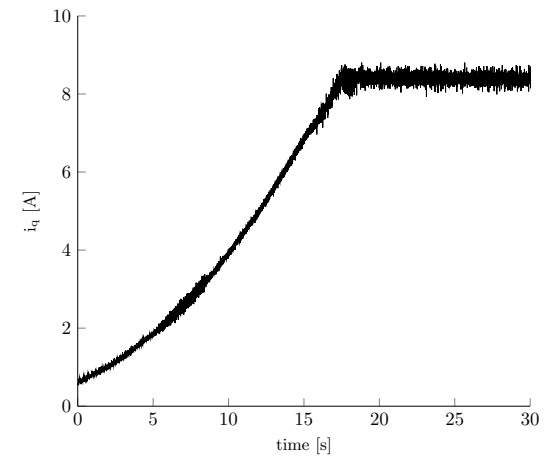

(b)

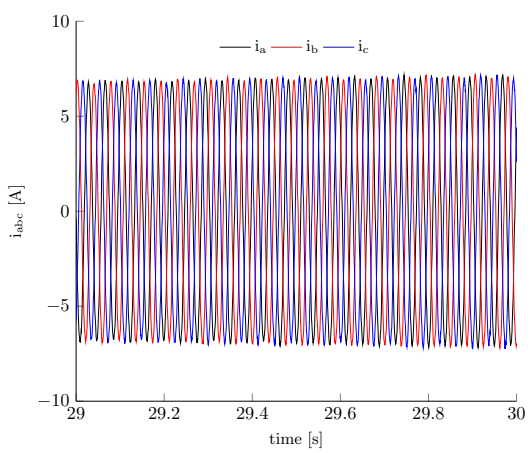

(d)

Fig. 9. Measured $i_{\mathrm{D}, \mathrm{Q}}$ and phase currents during the experiments of Fig. 8, with acceleration feedforward.

efficiency gap reduces, while the PM cost increases. Nevertheless, other factors, as those mentioned above (cabling, cooling, well drilling, etc.), along with the prescriptions coming from international eco-directives, are going to make the PMSM and its anisotropic variants (up to pure synchronous reluctance motors) a viable solution also at higher power ratings.

From the software perspective, the control of the pumping system to ensure an efficient MPPT and maximise the energy extraction is usually IP protected by manufacturers, and little information is usually available. The contribution in this paper is a verified and effective MPPT strategy, based on a modification of the well-known FOC using extremum seeking control. The resulting control system has minimum complexity, easy tuning and can be quickly developed and embedded also in commercial solutions.

\section{REFERENCES}

[1] R. Barlow, B. McNelis, and A. Derrick, "Solar Pumping; An Introduction and Update on the Technology, Performance, Costs and Economics," World Bank - Technical Papers, Working Papers, 1993.

[2] M. Z. Youssef, "Design and performance of a cost-effective BLDC drive for water pump application," IEEE Transactions on Industrial Electronics, vol. 62, no. 5, pp. 3277-3284, May 2015.

[3] S. Makukatin, "Water from the African sun," Spectrum, IEEE, vol. 31 , no. 10 , pp. $40-43$, Oct. 1994.

[4] G. Terorde, K. Hameyer, and R. Belmans, "Sensorless control of a permanent magnet synchronous motor for PV-powered water pump systems using the extended Kalman filter," in Electrical Machines and Drives, 1999. Ninth International Conference on (Conf. Publ. No. 468), 1999, pp. 366-370.

[5] L. An and D. D. C. Lu, "Design of a single-switch DC/DC converter for a PV-battery-powered pump system with PFM+PWM control," IEEE Transactions on Industrial Electronics, vol. 62, no. 2, pp. 910-921, Feb 2015.
[6] M. A. Vitorino, M. B. de Rossiter Correa, C. B. Jacobina, and A. M. N. Lima, "An effective induction motor control for photovoltaic pumping," IEEE Transactions on Industrial Electronics, vol. 58, no. 4, pp. 11621170, April 2011.

[7] J. M. Caracas, G. D. C. Farias, L. M. Teixeira, and L. de Souza Ribeiro, "Implementation of a High-Efficiency, High-Lifetime, and Low-Cost Converter for an Autonomous Photovoltaic Water Pumping System," Industry Applications, IEEE Transactions on, vol. 50, no. 1, pp. 631641, Jan.-Feb. 2014.

[8] S. Jain, R. Karampuri, and V. T. Somasekhar, "An integrated control algorithm for a single-stage PV pumping system using an open-end winding induction motor," IEEE Transactions on Industrial Electronics, vol. 63, no. 2, pp. 956-965, Feb 2016.

[9] B. Subudhi and R. Pradhan, "A comparative study on maximum power point tracking techniques for photovoltaic power systems," Sustainable Energy, IEEE Transactions on, vol. 4, no. 1, pp. 89-98, Jan 2013.

[10] D. Sera, R. Teodorescu, J. Hantschel, and M. Knoll, "Optimized maximum power point tracker for fast-changing environmental conditions," IEEE Transactions on Industrial Electronics, vol. 55, no. 7, pp. 26292637, July 2008.

[11] M. A. G. de Brito, L. Galotto, L. P. Sampaio, G. d. A. e Melo, and C. A. Canesin, "Evaluation of the main MPPT techniques for photovoltaic applications," IEEE Transactions on Industrial Electronics, vol. 60, no. 3, pp. 1156-1167, March 2013.

[12] Y. Wang, Y. Li, and X. Ruan, "High-accuracy and fast-speed MPPT methods for PV string under partially shaded conditions," IEEE Transactions on Industrial Electronics, vol. 63, no. 1, pp. 235-245, Jan 2016.

[13] R. Leyva, C. Alonso, I. Queinnec, A. Cid-Pastor, D. Lagrange, and L. Martinez-Salamero, "MPPT of photovoltaic systems using extremum - seeking control," Aerospace and Electronic Systems, IEEE Transactions on, vol. 42, no. 1, pp. 249-258, Jan 2006.

[14] R. Leyva, P. Artillan, C. Cabal, B. Estibals, and C. Alonso, "Dynamic performance of maximum power point tracking circuits using sinusoidal extremum seeking control for photovoltaic generation," International Journal of Electronics, vol. 98, no. 4, pp. 529-542, 2011.

[15] S. Brunton, C. Rowley, S. Kulkarni, and C. Clarkson, "Maximum Power Point Tracking for Photovoltaic Optimization Using Ripple-Based Extremum Seeking Control," Power Electronics, IEEE Transactions on, vol. 25 , no. 10 , pp. 2531-2540, Oct 2010. 
[16] F. Castaos and C. Kunusch, "Ditherless extremum seeking for hydrogen minimization in PEM fuel cells," IEEE Transactions on Industrial Electronics, vol. 62, no. 8, pp. 5218-5226, Aug 2015.

[17] L. Fu and U. Ozguner, "Extremum-seeking control in constrained source tracing with nonholonomic vehicles," IEEE Transactions on Industrial Electronics, vol. 56, no. 9, pp. 3602-3608, Sept 2009.

[18] T. Esram, J. Kimball, P. Krein, P. Chapman, and P. Midya, "Dynamic maximum power point tracking of photovoltaic arrays using ripple correlation control," Power Electronics, IEEE Transactions on, vol. 21, no. 5, pp. 1282-1291, Sept 2006.

[19] M. Villalva, J. Gazoli, and E. Filho, "Comprehensive approach to modeling and simulation of photovoltaic arrays," Power Electronics, IEEE Transactions on, vol. 24, no. 5, pp. 1198-1208, May 2009.

[20] C. Zhang, A. Siranosian, and M. Krstić, "Extremum seeking for moderately unstable systems and for autonomous vehicle target tracking without position measurements," Automatica, vol. 43, no. 10, pp. 1832 - 1839, 2007. 